\section{(6) OPEN ACCESS}

\title{
Oral versus intravenous iron replacement therapy distinctly alters the gut microbiota and metabolome in patients with IBD
}

\author{
Thomas Lee, ${ }^{1,2}$ Thomas Clavel, ${ }^{3}$ Kirill Smirnov, $^{4}$ Annemarie Schmidt, ${ }^{5}$ \\ Ilias Lagkouvardos, ${ }^{3}$ Alesia Walker, ${ }^{4}$ Marianna Lucio, ${ }^{4}$ Bernhard Michalke, ${ }^{4}$ \\ Philippe Schmitt-Kopplin, ${ }^{3,4}$ Richard Fedorak, ${ }^{1}$ Dirk Haller ${ }^{3,5}$
}

\begin{abstract}
- Additional material is published online only. To view please visit the journal online (http://dx.doi.org/10.1136 gutjnl-2015-309940).

For numbered affiliations see end of article.
\end{abstract}

Correspondence to Professor Dirk Haller, Chair of Nutrition and Immunology, Technische Universität München, Gregor-Mendel-Str. 2, Freising 85354, Germany; dirk. haller@tum.de

TL, TC and KS contributed equally and share first authorship.

$\mathrm{RF}$ and DH contributed equally and share last authorship.

Received 9 May 2015 Revised 2 December 2015 Accepted 21 December 2015 Published Online First 4 February 2016
CrossMark

To cite: Lee T, Clavel T,

Smirnov K, et al.

Gut 2017;66:863-871.

\section{ABSTRACT}

Objective Iron deficiency is a common complication in patients with IBD and oral iron therapy is suggested to exacerbate IBD symptoms. We performed an openlabelled clinical trial to compare the effects of per oral (PO) versus intravenous (IV) iron replacement therapy (IRT).

Design The study population included patients with Crohn's disease (CD; $N=31), U C(N=22)$ and control subjects with iron deficiency (non-inflamed, $\mathrm{NI}=19$ ). After randomisation, participants received iron sulfate (PO) or iron sucrose (IV) over 3 months. Clinical parameters, faecal bacterial communities and metabolomes were assessed before and after intervention.

Results Both PO and IV treatments ameliorated iron deficiency, but higher ferritin levels were observed with IV. Changes in disease activity were independent of iron treatment types. Faecal samples in IBD were characterised by marked interindividual differences, lower phylotype richness and proportions of Clostridiales. Metabolite analysis also showed separation of both UC and CD from control anaemic participants. Major shifts in bacterial diversity occurred in approximately half of all participants after IRT, but patients with CD were most susceptible. Despite individual-specific changes in phylotypes due to IRT, PO treatment was associated with decreased abundances of operational taxonomic units assigned to the species Faecalibacterium prausnitzii, Ruminococcus bromii, Dorea sp. and Collinsella aerofaciens. Clear IV-specific and PO-specific fingerprints were evident at the level of metabolomes, with changes affecting cholesterol-derived host substrates.

Conclusions Shifts in gut bacterial diversity and composition associated with iron treatment are pronounced in IBD participants. Despite similar clinical outcome, oral administration differentially affects bacterial phylotypes and faecal metabolites compared with IV therapy.

Trial registration number clinicaltrial.gov (NCT01067547).

\section{INTRODUCTION}

Iron deficiency anaemia is a common clinical issue in patients with IBD, and iron replacement therapy (IRT) improves anaemia and is associated with improved quality of life. ${ }^{1}$ Conflicting results about

\section{Significance of this study}

What is already known on this subject?

- The high prevalence of iron deficiency anaemia in patients with IBD requires therapeutic intervention based on oral or intravenous (IV) iron application.

- Oral iron treatment is standard, but GI side effects and the potency to exacerbate intestinal inflammation support clinical implementation of IV iron therapy.

- High concentrations of luminal iron affect the gut microbiota composition and disease activity in IBD.

What are the new findings?

- Bacterial communities are most sensitive to iron therapy in patients with Crohn's disease (CD).

- Oral and IV iron therapies differentially affect bacterial communities and the metabolic landscape in patients with IBD.

- Shifts in faecal metabolome and bacterial communities are dissociated from changes in disease activity in patients with IBD.

How might it impact on clinical practice in the foreseeable future?

- IV iron therapy might specifically benefit anaemic patients with $C D$ with an instable microbiota.

clinical outcome regarding the route of IRT have been published. ${ }^{3}$ Total iron replacement is possible with intravenous (IV) iron infusions considered to be safe and efficacious. ${ }^{4}$ With regard to oral iron, it has been advocated that lower doses of oral iron should be used as it is efficacious with less side effects.

Iron is a critical nutrient for the growth of microorganisms, and the tight regulation of intravascular iron availability forms part of the innate immune defence. ${ }^{5}$ However, similar iron regulation has not been elucidated in the intraluminal gut environment whereby many gut bacteria are competing for the free iron to maintain their growth. Most of the pathogenic bacteria have enhanced 
iron acquisition mechanisms contributing to increased virulence. $^{6}{ }^{7}$ This was demonstrated in a study involving African children where an increase in enterobacteria and a reduction in lactobacilli accompanied by increased faecal calprotectin levels were noted after 6 months of iron fortified biscuits. ${ }^{8}$ Most recently, Jaeggi et $a l^{9}$ confirmed the effect of iron fortification in Kenyan infants clearly demonstrating changes in faecal bacterial community structure associated with the selection of pathogens.

IBD is a result of disordered immune responses to commensal and pathogenic gut microbes in genetically susceptible individuals. ${ }^{10}$ Diversion of faecal stream in the setting of an ileostomy improves colonic inflammation, whereas re-establishment of the faecal stream causes recurrence of disease, clearly demonstrating the critical role of gut microbes in IBD pathogenesis. ${ }^{11} 12$ There is accumulating evidence available implicating the role of gut microbial dysbiosis in the pathogenesis and perpetuation of IBD; however, environmental factors promoting changes in the gut microbial ecosystem are poorly defined. ${ }^{13-16}$

We previously showed that dietary iron alters dominant gut bacterial communities and exacerbates chronic ileal inflammation in mice. ${ }^{17}$ However, as correctly noted in a recent commentary by Guenter Weiss, the relevance of these findings still awaits proof of concept in humans. ${ }^{18}$ Hence, we designed and conducted an open-labelled clinical trial with iron-deficient participants with or without IBD and performed pre-iron therapy and post-iron therapy measurements where the individuals served as their own controls. We measured faecal bacterial communities by high-throughput sequencing and metabolite profiles by high-resolution mass spectrometry. Clinical data included changes in serum haemoglobin, ferritin and iron saturation and the effect of iron therapy on the IBD status as captured by clinical disease activity index and $\mathrm{C}$ reactive protein (CRP).

\section{METHODS}

\section{Cohort and study design}

Participants were identified by their treating gastroenterologists and recruited during 2010 from the gastroenterology outpatient clinics in Edmonton (University of Alberta Hospital (UAH), Royal Alexandra Hosptial (RAH)). Written inform consent was obtained prior to web-based randomisation. Inclusion criteria were age $>18$ years and confirmed diagnosis of IBD by standard radiology, endoscopy and histopathology findings. The control group consisted of participants with a recent upper GI bleeding such as peptic ulcer disease, with a deliberate low iron diet (vegetarian), or women with non-specific GI symptoms and menorrhagia. All subjects were iron deficient, that is, iron saturation $<16 \%$ with or without ferritin $<30 \mu \mathrm{g} / \mathrm{L}$ (if normal CRP) or $<100 \mu \mathrm{g} / \mathrm{L}$ (if elevated CRP). Exclusion criteria included iron supplementation, blood transfusion or the use of antibiotics within the last 3 months prior to inclusion, untreated concurrent folate and/or vitamin $\mathrm{B}_{12}$ deficiency, history of intolerance to IRT, coeliac disease, known malignancy other than skin cancer and primary haematological disorder. Treatment allocation was 1:1 to either IV or per oral (PO) iron therapy. PO participants took one $300 \mathrm{mg}$ iron sulfate tablet twice a day. IV patients received three or four separate iron sucrose $300 \mathrm{mg}$ infusions if iron deficient only or with anaemia, respectively. Participants were followed up for 12 weeks from the initiation of IRT. The experimental design is summarised in figure $1 \mathrm{~A}$. A review of participants' clinical disease activity status at 12 months from the iron therapy initiation was performed with special emphasis on the need for therapeutic escalation (step-up medical or surgical therapy).

\section{Samples}

All participants were reviewed at baseline and 3 months after initiating IRT. At these time points, serum and stool specimens were collected. All participants were given a stool collection kit with a pictorial instruction on how to collect stools specimens and minimise contamination. Stool samples were frozen within $30 \mathrm{~min}$ and kept at $-80^{\circ} \mathrm{C}$ until further processing for sequencing and mass spectrometry analysis. We can thus exclude confounding effects of freezing/thawing cycles or storage at $-20^{\circ} \mathrm{C}$ over long periods of time. As the recruitment happened over 18 months, some specimens were kept frozen longer than others. However, this was the case for samples across all groups that were compared, precluding group-specific artefact observations due to the sampling/storage procedure. Quality of life was assessed using Shorten IBD Questionnaire (SIBDQ) and the Euro Quality 5 Dimensions Visual Analogue Scale (EQ5D VAS). At 3 months, subjects were reviewed for medication adherence and possible adverse event. IBD activity was assessed with Modified Harvey Bradshaw Index (HBI) for Crohn's disease (CD) and Partial Mayo (pMayo) score for UC, in conjunction with serum CRP. Adherence to PO iron therapy was assessed by pill count at week 12 and adherence to IV iron was determined by attendance at the infusion clinic. The disease activity and clinical course of the IBD cohort were also reviewed at 12 months after initiation of treatment.

\section{High-throughput 16S rRNA gene sequencing}

Samples were processed and data were analysed as described previously. ${ }^{19}$ Detailed information is provided in the online supplementary methods.

\section{High-resolution mass spectrometry and metabolome analysis}

Samples were measured via Fourier transform ion cyclotron resonance mass spectrometer (FT-ICR-mass spectrometry (MS)) and acquired data were processed by in-house developed pipelines as described in the online supplementary methods.

\section{Iron measurement}

The procedure for quantification of iron content in faeces is detailed in the online supplementary methods.

\section{Statistical analysis}

All data sets, including operational taxonomic unit (OTU) counts and abundances of taxonomic groups as well as metabolome data, are provided in online supplementary table S1. Statistical analyses were performed in the R programming environment or using SIMCA-P+12 (Umetrics).

Sequencing data were analysed statistically as published previously. ${ }^{19}$ Prior to testing for variations in OTU or taxonomic counts, individual counts $<0.5 \%$ were zeroed, and only prevalent and dominant taxonomic groups or molecular species (ie, detected in at least $30 \%$ of the participants $(n=124)$ and with a median sequence abundance $>1 \%$ in at least one group) were considered.

For metabolome analysis, data were autoscaled prior to applying any multivariate tools. Feature selection for a given classification was done according to the absolute values of regression coefficients or variable importance in projection (VIP score) derived from applying orthogonal partial least squares discriminant analysis (OPLS-DA). In order to estimate univariate significance of the selected features, $p$ values were calculated using the Mann-Whitney test. After selecting an adequate set of features (per extension, metabolites), OPLS-DA was reperformed using 
Figure 1 Clinical design and outcomes. (A) Anaemic subjects diagnosed with IBD and control anaemic participants without GI disorders were enrolled in the study and randomised into the oral or intravenous iron replacement therapy (IRT) group. Faecal samples were collected at baseline and after the end of intervention (3 months later).

Samples were analysed by high-throughput $16 \mathrm{~S}$ rRNA gene sequencing and Fourier transform ion cyclotron resonance mass spectrometer (FT-ICR-mass spectrometry (MS)) for analysis of bacterial communities and metabolites, respectively. (B) IRT restores iron storage, independently of the route of administration. (C) IBD activity was assessed with Modified Harvey Bradshaw Index (HBI) for CD and Partial Mayo (pMayo) score for UC. Values are $\%$ changes. (D) $\%$ changes in quality of life per participants category. Quality of life was scored according to Shorten IBD Questionnaire (SIBDQ) and Euro Quality 5 Dimensions Visual Analogue Scale (EQ5D). CD, Crohn's disease; CRP, C reactive protein; EQ5D, Euro Quality 5 Dimensions; IV, intravenous; $\mathrm{NI}$, non-IBD control anaemic subjects; PO, per oral; SIBDQ, Shorten IBD Questionnaire.
A

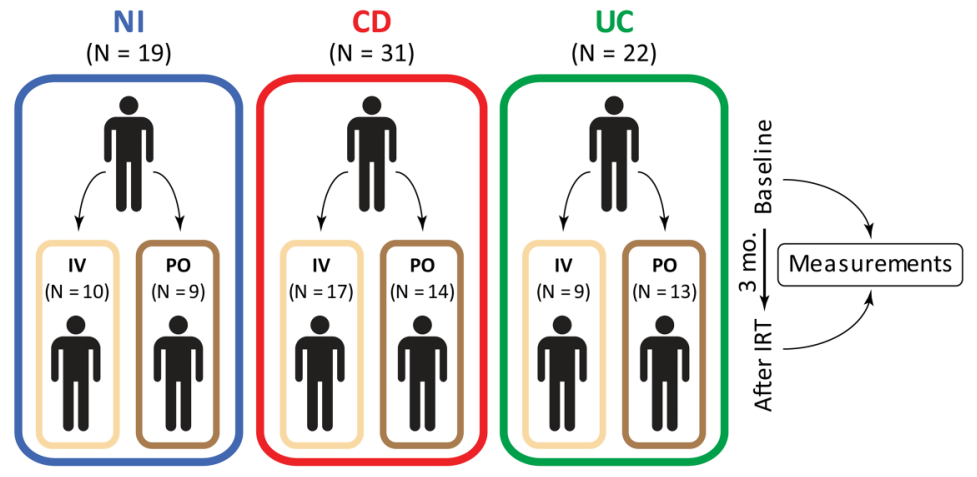

B
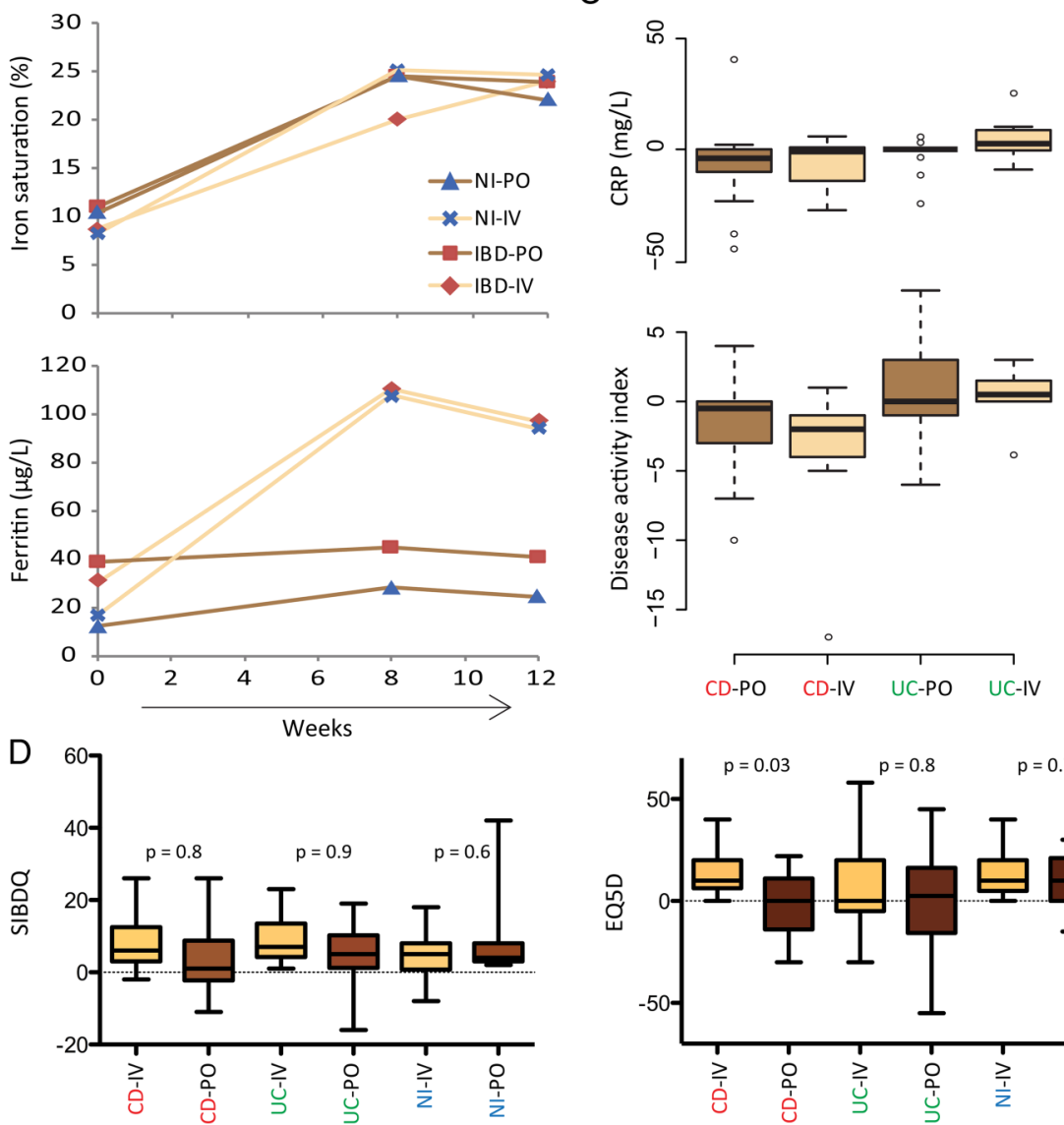

sevenfold cross-validation and the quality of the model was checked by the values of $R^{2} Y$ and $Q^{2} C U M$ (goodness of fit and predictive ability, respectively).

\section{RESULTS}

IRT restores iron storage, independently of the administration route

A total of 72 participants completed the study (36 in each of the PO and IV group), including 19 non-inflamed (NI) controls, 31 patients with $C D$ and 22 patients with UC. After intervention, the mean iron saturation level in the control group was no different between the PO route $(23.3 \pm 6.3 \%)$ and IV route $(23.9 \pm 9.9 \%)$. Comparable mean iron saturation was achieved at 3 months in the IBD cohort: PO route $(24.7 \pm 15.8 \%)$ and IV route $(24.2 \pm 13.4 \%)$ (figure $1 \mathrm{~B})$. A statistically significantly higher serum ferritin level at 3 months was noted in the IV cohort compared with PO in both NI and IBD participants (figure 1B). The route of IRT did not affect haemoglobin levels at 3 months (data not shown).
Impact of iron treatment on disease activity and quality of life

The route of IRT did not affect disease activity based on changes in the clinical disease activity indices (MHBI and pMayo) and serum concentrations of CRP (figure 1C). At 12 months follow-up, $37 \%$ of the PO $(n=27)$ and $30 \%$ of the IV $(n=124)$ cohort required therapeutic escalation requiring biological therapy or surgery $(p=0.63)$. Overall, there was a trend towards higher magnitude of improved SIBDQ scores in IBD-IV groups, but results did not reach significance (figure 1D). In contrast, the VAS (EQ5D VAS) indicated improvement in CD participants on the IV treatment (figure 1D), suggesting that IV iron may be associated with a better quality of life in CD. With respect to adverse events, one CD participant in the IV group developed arterial thrombosis of the left leg requiring below knee amputation. Other adverse events included generalised arthralgia and headaches. In the PO cohort, one participant had self-limiting nausea. No adverse event was reported in the control group. Adherence to IV iron therapy was $100 \%$ in all 
participants. The overall adherence rate in the PO cohort ranged from taking $85-100 \%$ of the iron tablets.

\section{IBD is linked to specific faecal microbiota and metabolome fingerprints}

After sequence processing and filtering, we obtained a total of 6726192 chimera-checked 16S rRNA gene sequences (53 382 \pm 13153 per sample) spanning a total of 372 OTUs $(121 \pm 37$ per sample). We first looked at the presence of IBD-specific differences in bacterial diversity and composition by analysing all samples independently of the time point of measure. Multidimensional scaling revealed a significant clustering of samples according to the occurrence of IBD (figure 2A). Both IBD groups were characterised by marked interindividual differences in 16S rRNA profiles when compared with NI participants, who represented a more homogenous group of samples. CD participants were most distinguishable from controls, without specific subclustering according to disease state, that is, patients in an active phase of the disease or in remission were scattered across the clusters. Analysis of Shannon effective diversity showed that IBD subjects were characterised by a significant drop in taxa richness, and this was most pronounced in patients with $\mathrm{CD}$, who showed a median effective count $<15$ species (figure 2B). To see whether differences observed at the level of diversity were associated with changes in the relative abundance of specific bacteria, we looked for significant differences in sequence proportions of taxonomic groups from phyla down to families. Firmicutes was the most dominant phylum in the samples analysed, followed by Actinobacteria and Bacteroidetes. Members of the latter phylum were completely absent in samples from patients with UC in the active state of disease (see online supplementary figure S1A). Among Firmicutes, we observed a drop in the relative abundance of Clostridiales in patients with $\mathrm{CD}$ (figure 2C). At the family level, higher median abundance of Christensenellaceae was specific for NI subjects. Within the phylum Actinobacteria, Bifidobacteriaceae showed higher abundances in patients with IBD (figure 2C), which might be related to dietary intake of probiotics and prebiotics in the form of commercially available yogurts in 10 participants.

With respect to metabolomics analysis, $7578 \mathrm{~m} / \mathrm{z}$ values out of the original 204445 features detected (ca. 4\%) passed filtering, of which $2260 \mathrm{~m} / \mathrm{z}$ values were annotated as putative metabolites without differentiation between human, plant or bacterial origin. An OPLS-DA model based on 1012 features revealed that baseline faecal samples from patients without IBD separated well from patients with CD (figure 2D). Of the $300 \mathrm{~m} / \mathrm{z}$ values assigned to putative metabolites, 13 were most discriminative for classification of the CD and NI groups, including higher signal intensities for ceramide phosphate and three fatty acids in faeces from patients with CD (figure 2E). The OPLS-DA model built using the samples belonging to NI and UC classes at baseline was less robust than the model discriminating NI and CD classes, with $\mathrm{R}^{2} \mathrm{Y}$ and $\mathrm{Q}^{2} \mathrm{CUM}$ values of 0.3 and 0.21 , respectively (figure $2 \mathrm{~F}$ ). Of the $331 \mathrm{~m} / \mathrm{z}$ values assigned to putative metabolites, 13 were most discriminative, including lower intensities of phospholipids in patients with UC (figure $2 \mathrm{G}$ ).

\section{Faecal metabolomes and bacterial communities are prone to instability in anaemic IBD versus NI patients following IRT}

We next looked at variations of bacterial communities and metabolomes in faeces when discriminating samples before and after IRT. Multidimensional analysis showed that the effect of treatment overtime did not overrule IBD-specific clustering, that is, we did not observe major effects of oral or IV iron on bacterial diversity that would generate treatment-specific clusters of samples (figure 3A). Changes were individual specific, that is, distances between samples before and after treatment ranged from short to extreme, suggesting that faecal bacterial communities from several subjects are differentially sensitive to changes during IRT. Comparison of phylogenetic distances demonstrated that paired samples (before and after therapy for each individual) were more distantly related in patients with CD and patients with UC than in control anaemic participants, indicating that faecal bacterial communities in IBD subjects are more sensitive to IRT-induced alterations (figure 3B). Dendrogram analysis illustrated that patients with CD (red colour in the outer ring) had the most distinct diversity, but most of all that these patients were characterised by the lowest number of paired samples, defined as two samples from one given individual being most closely related to each other than to any other sample in the data set (black boxes) (figure 3C). When classifying subjects according to changes in the disease status (improved, unchanged, worsen) on the basis of clinical scores and CRP levels, and independently of IBD type (CD or UC), a decrease in sequence proportions of members of the family Ruminococcaceae was observed in subjects with improvement of disease (see online supplementary figure S1B).

To assess the impact of IRT on faecal metabolome, we looked at the sample arrangement in a low-dimensional space produced by OPLS-DA for differentiation between NI controls and patients with CD (prediction for NI and UC subjects after IRT was not performed since the corresponding model was weak) (figure 3D). This analysis showed that sample points from the NI and CD groups after IRT (triangles) grouped together compared with sample clouds at baseline (circles), which showed divergence between NI and CD. This indicates that, in comparison with clear CD-specific features at baseline, faecal metabolomes converged after IRT.

\section{The gut ecosystem is affected by oral iron treatment}

Comparing samples at baseline and after IRT taking into account the route of administration independently of disease phenotype, the iron content in faeces was increased after PO $(p=0.014)$ but not after IV $(p=0.363)$ treatment (paired $t$ test) (figure 4A). In contrast, total sulfur concentrations were not significantly different. Because of these changes affecting iron levels in faeces, we assessed whether the route of iron administration influenced changes in bacterial communities and metabolite landscape.

Independently of disease activity phenotype, we searched for differences between PO and IV treatment when considering delta-values calculated as differences in sequence abundances (after-before). We found only five single OTUs that showed differential effects, four of which had lower abundances after oral iron therapy (figure 4B). These OTUs were identified as Collinsella aerofaciens (OTU-6), Faecalibacterium prausnitzii (OTU-13), Ruminococcus bromii (OTU-23) and Dorea sp. (OTU-52). In contrast, one OTU belonging to the genus Bifidobacterium (OTU-620) was found at higher relative abundances in faeces from subjects treated with iron orally, although four participants in this group consumed prebiotics and probiotics versus six in the IV group.

An OPLS-DA classification model of the routes of administration independently of disease phenotype was built to find treatment-specific differences in faecal metabolomes and showed that IV and PO grouped distinctly (figure 4C). A total of 1040 features were used to build the model and 308 of them 
Figure 2 IBD-specific features of faecal microbiota and metabolomes. (A) NMDS plot based on generalised UniFrac distances, including all patients before and after iron replacement therapy, indicating that bacterial communities in patients with $C D$ are most heterogeneous and distant from UC and control subjects. (B) Shannon effective diversity boxplots display decreased numbers of dominant molecular species in patients with IBD. (C) Relative abundances of dominant bacterial taxa with significant difference between subject categories. The numbers of samples, in which the given bacteria were detected, are shown in brackets below categories. (D) Score plot of orthogonal partial least squares discriminant analysis (OPLS-DA) classification of non-inflamed (NI) control anaemic participants (blue circles) and patients with $\mathrm{CD}$ (red circles) at baseline. Learning the model resulted in one predictive and one orthogonal component ( $\mathrm{x}$-axis and $y$-axis, respectively). (E) Top features mostly responsible for classification in the corresponding OPLS-DA model in (D). The features were chosen according to the values of variable importance in projection scores. $P$ values obtained by Mann-Whitney test are shown. (F) Score plot of OPLS-DA classification of $\mathrm{NI}$ control anaemic participants (blue bars) and patients with UC (green bars) at baseline. Learning the model resulted in only one predictive component (x-axis). The $y$-axis indicates individual samples in each group. (G) Top features mostly responsible for classification in the corresponding OPLS-DA model in (F). ANOVA, analysis of variance; $B$, baseline; CD, Crohn's disease; NI, non-IBD control anaemic subjects; NMDS, non-metric multidimensional scaling. ${ }^{*}<0.05 ;{ }^{* * *}<0.01$.
A

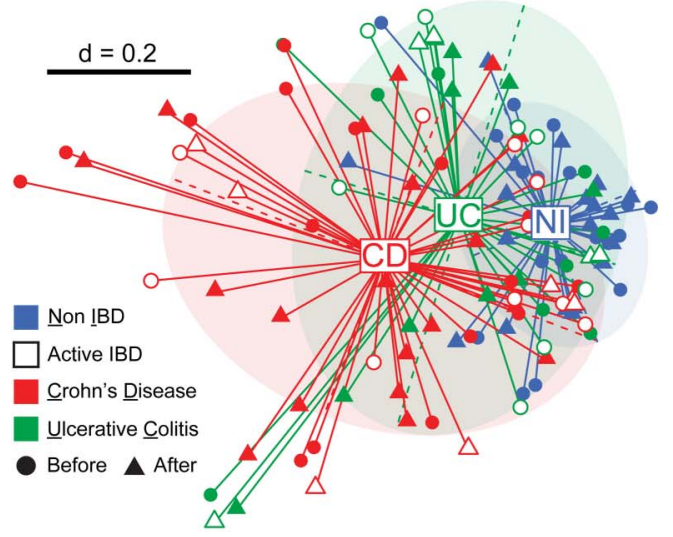

C
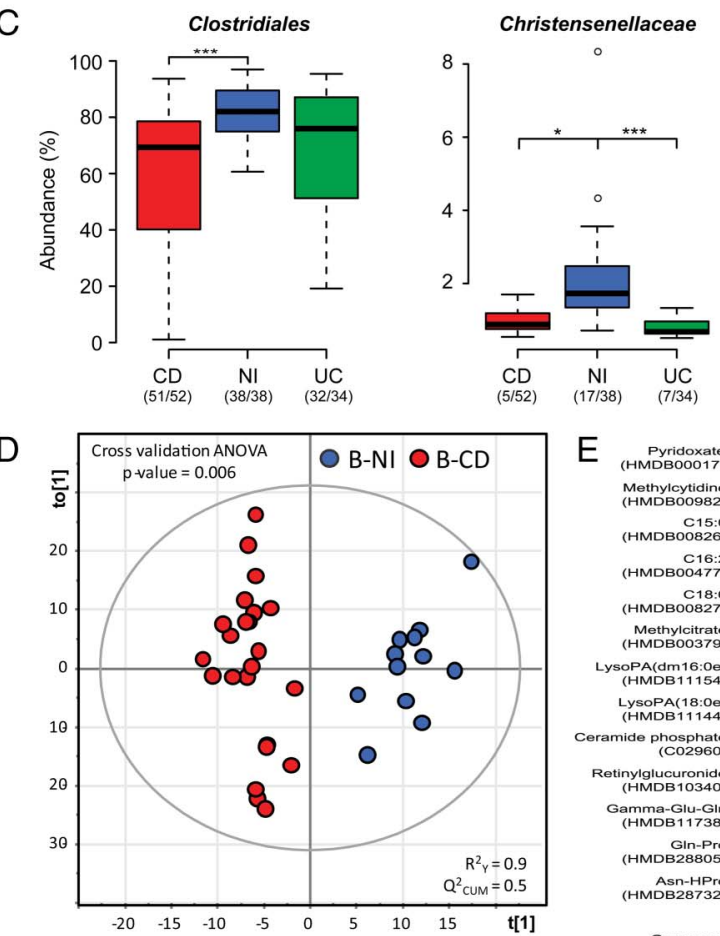

F

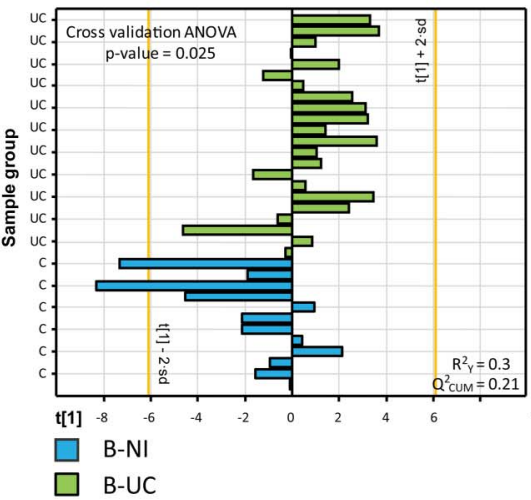

B

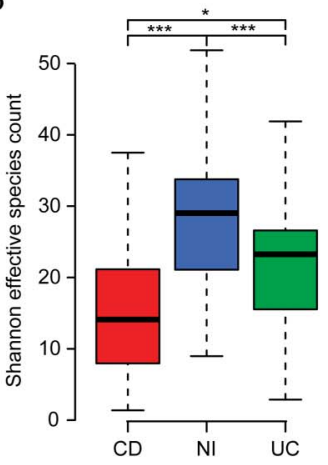

Bifidobacteriaceae

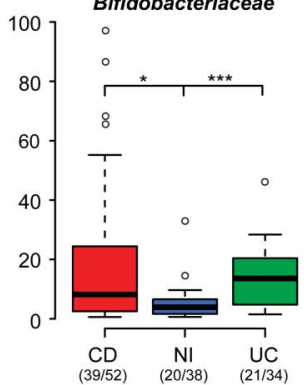

$E_{\substack{\text { Pridoxato } \\ \text { (HMDBO0017) }}}$ Methylcytidine
(HMDBO0982) C15:0
(HMDBO0826) C16:2 (HMDBоO827) Methylcitrate
(HMDBO0379) LysoPA(dm16:0e)
(HMDB11154) LysoPA(18:0e)
(HMDB11144)

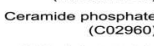
Retinylglucuronide
(HMDB10340) Gamma-Glu-GIn
(HMDB11738) (HMDB28805) (HMDB28732)

\section{$\mathrm{G}$}

Cervonoy!

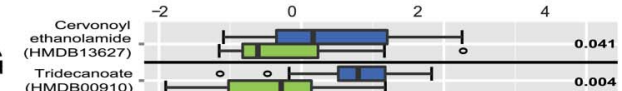
Tridecanoate
(HMDBO0910) Ocotetradecanoate
(HMDB10730) (NH) sulfanyloctanoate
(HMDB13639) Undecanedioato
(HMDBO0888) Sebacate
(HMDBO0792) Dodecanedioate LYSOPE (16:0 (HMSOPE(15:0) LYSoPE (14:0) Carboxytocopherol

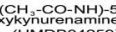
Tyr-HPro
(HMDB29106) (HMDB29106) were assigned to putative metabolites via the MassTRIX web server. The top-17 metabolites responsible for separation of IV and PO groups together with the output of univariate statistics are shown in figure 4D. Metabolites displaying higher intensities in the PO group included phosphatidylglycerol, palmitate and derivatives thereof, whereas bile acids, steroids (tetrahydrodeoxycorticosterone) and other cholesterol derivatives were characteristics of the IV group.

\section{DISCUSSION}

The high prevalence of iron deficiency anaemia in IBD requires therapeutic intervention based on oral IRT or IV-IRT. ${ }^{20}$ Oral IRT has long been considered as standard intervention based on the safety profile, low cost and convenient administration. However, the high frequency of GI side effects ${ }^{21}$ and the potency to exacerbate intestinal inflammation support clinical implementation of IV-IRT. ${ }^{22}{ }^{23}$ We previously showed that the 
A

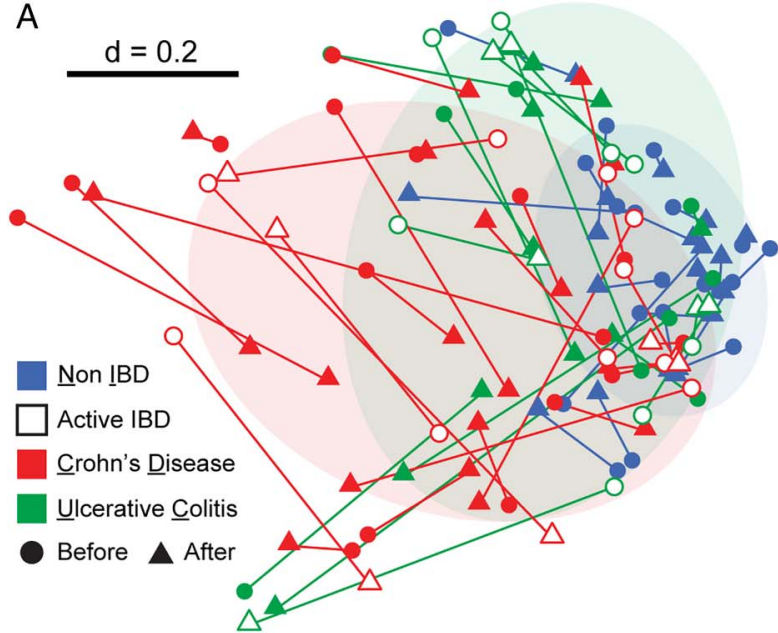

B

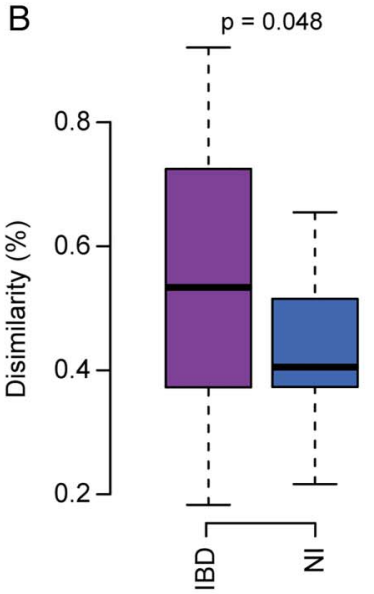

C
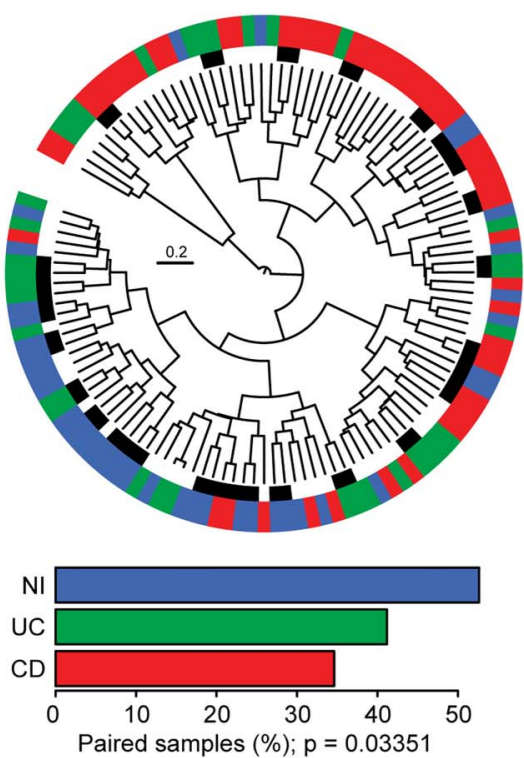

D

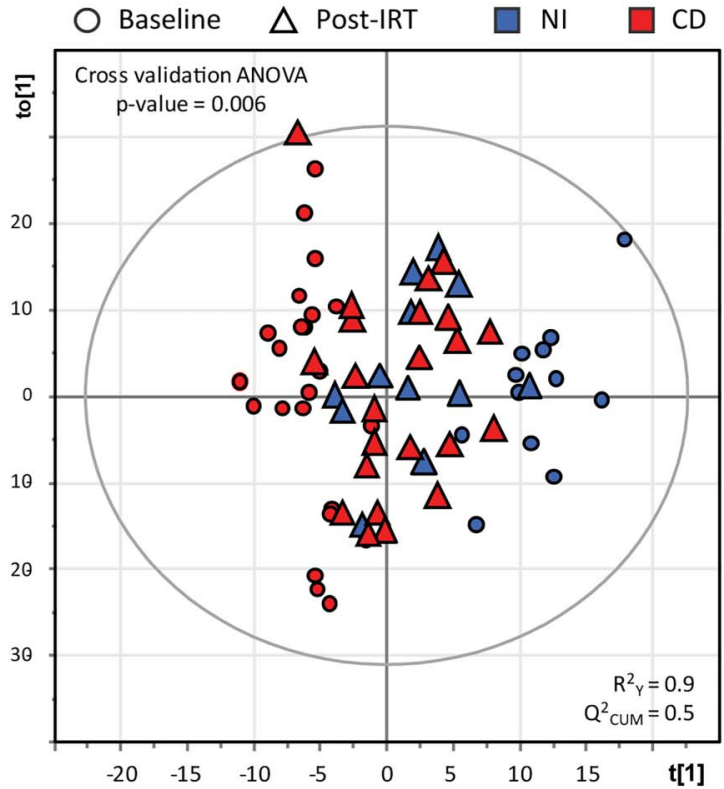

Figure 3 The gut environment in patients with IBD versus control anaemic subjects is more sensitive to overtime changes associated with iron replacement therapy (IRT). (A) NMDS plot of phylogenetic distances showing overall changes in bacterial diversity before and after treatment (connecting lines). (B) Boxplots depict the distribution of generalised UniFrac distances per patient category, indicating that samples before and after IRT are more similar in non-inflamed (NI) control subjects than in patients with IBD. (C) Wards' clustering dendrogram calculated from phylogenetic distances between all samples. Paired samples (located next to each other before and after IRT for one given individual) are marked with black bars (inner ring). Disease categories were colour-coded in the outer ring as follows: red, Crohn's disease (CD); green, UC and blue, NI. Percentages of paired samples per patient category are shown in the barplot. The $\mathrm{p}$ value was obtained using a $\chi^{2}$ test. (D) Influence of iron treatment on classification between control and CD participants. The position of points corresponding to the samples from after IRT (blue and red triangles) was predicted using the orthogonal partial least squares discriminant analysis (OPLS-DA) model built for classification of $\mathrm{NI}$ and CD samples at baseline (blue and red circles). ANOVA, analysis of variance; NMDS, non-metric multidimensional scaling.

absence of luminal ferrous iron was associated with major changes in the gut microbiota and the prevention of chronic inflammation in an experimental model for CD-like ileitis. ${ }^{17} 24$ To follow up on these findings, the aim of the present study was to investigate the impact of oral (PO) and IV iron treatment on the intestinal milieu via assessment of faecal bacterial diversity and composition as well as metabolome analysis in subjects with iron deficiency suffering from CD or UC.

In accordance with previous reports, ${ }^{25-28}$ distinct bacterial phylogenetic makeups and lower phylotype richness were observed in faeces from patients with IBD compared with NI control anaemic subjects. Most interesting regarding the impact of IRT, faecal bacterial communities in patients with $\mathrm{CD}$ were characterised by highest interindividual differences and were most sensitive to changes after 3 months of treatment. Despite limitations of faecal material for bacterial community analysis, ${ }^{29}$ the drop observed in the relative abundance of Clostridiales spp. is consistent with the most comprehensive analysis in treatment-naïve paediatric patients with $\mathrm{CD} .{ }^{25}$ In the latter study, changes in stool samples were actually representative for differences in biopsy material at the terminal ileum and rectum. This implies an important disease-related effect on members of the order Clostridiales independent of medication and sampling procedures. Specifically, reduction of Clostridium cluster IV and XIV members has been proposed to contribute to dysbiosis in patients with $\mathrm{CD},{ }^{30} 31$ and selected mixtures of corresponding clostridia strains were shown to induce Tregulatory cells with disease-suppressive phenotypes in IBD-related mouse models. ${ }^{32}$ These results support a potential protective role of certain Clostridiales spp. in IBD pathogenesis. In our study, members of the newly described bacterial family 

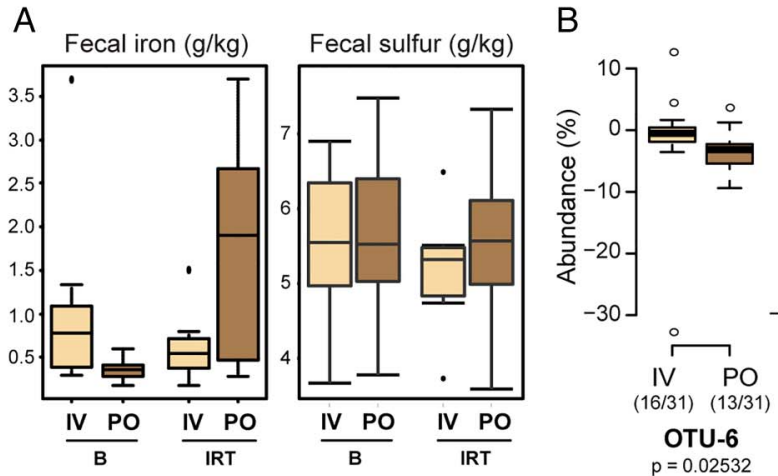

C

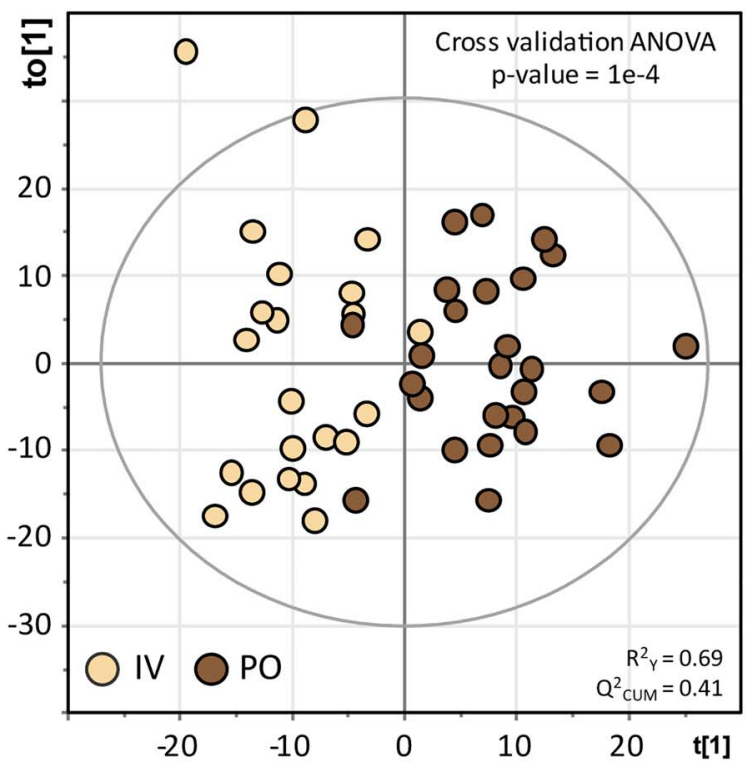

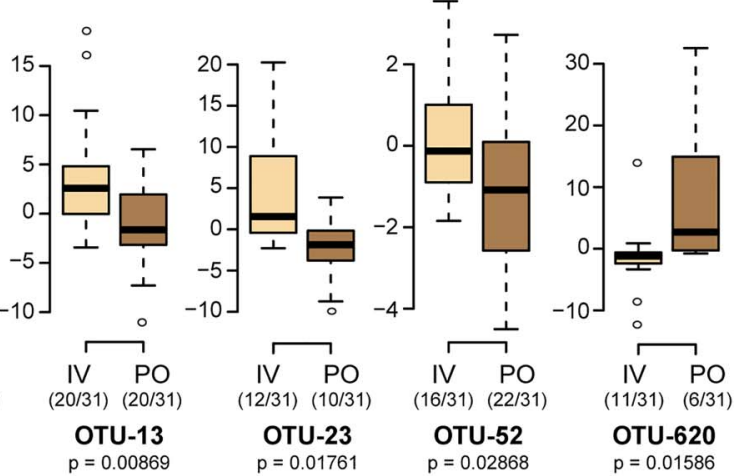

D

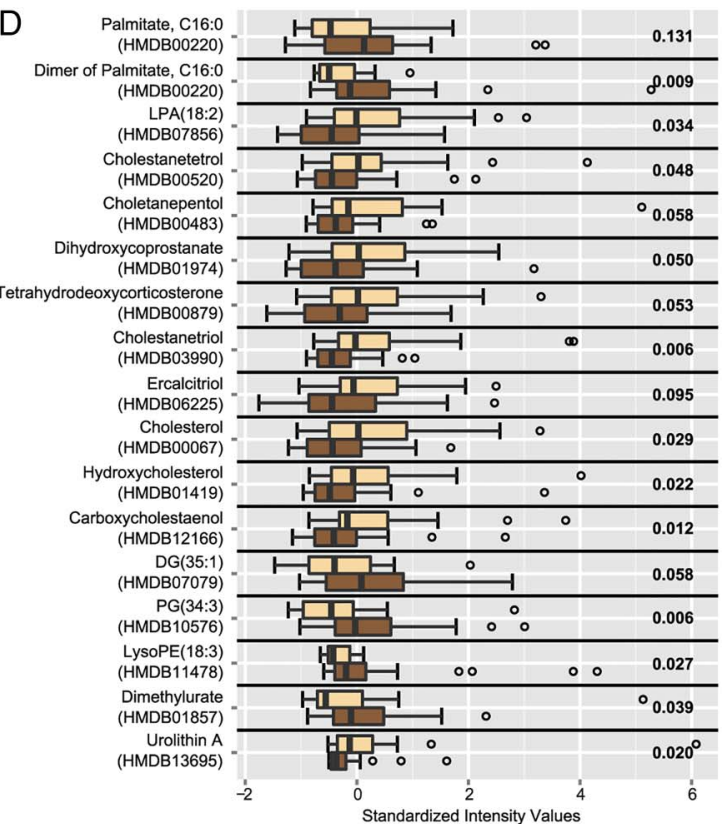

Figure 4 PO iron treatment is associated with specific features in faecal samples. (A) Iron and total sulfur content in faeces. (B) Relative abundance of phylotypes that reacted differently to intravenous (IV) or oral iron therapy. IRT groups were compared using Mann-Whitney test. Prior to testing, individual counts $<0.5 \%$ were zeroed, and only prevalent and dominant molecular species (ie, detected in at least $30 \%$ of the participants ( $n=124)$ and with a median sequence abundance $>1 \%$ in at least one group) were considered. (C) Score plot of orthogonal partial least squares discriminant analysis (OPLS-DA) classification of IV-IRT (beige) versus PO-IRT (brown). Learning the model resulted in one predictive and one orthogonal component (x-axis and y-axis, respectively). (D) Top features responsible for classification in the OPLS-DA model are shown as boxplots. $\mathrm{B}$, baseline; IRT, iron replacement therapy; OTU, operational taxonomic unit; PO, per oral.

Christensenellaceae were specific for NI conditions. ${ }^{33}$ However, other family members within the order Clostridiales such as Ruminococcaceae as well as the Veillonellaceae within the order Selenomonadales are rather increased in patients with IBD, ${ }^{25} 2834$ making general disease-related conclusions at these high taxonomic levels rather difficult.

The transition from a quiescent state to disease exacerbation in patients with IBD was shown to be associated with alterations of faecal bacterial communities, ${ }^{35}$ which supports the hypothesis that inflammatory processes in the intestines of both patients with UC and patients with CD affect the microbial milieu. In our study, IRT-induced shifts in bacterial diversity and composition in faeces from patients with IBD were not associated with changes in disease activity, suggesting that patient-specific shifts in faecal microbiota are not necessarily linked to changes in disease severity over short periods of intervention. Regarding the route of iron administration, IV therapy confirmed a slightly more effective management of iron deficiency anaemia compared with oral treatment with respect to serum ferritin levels. In addition and consistent with the presence of increased iron in faeces, the luminal milieu in faeces from PO-treated individuals was distinct at the level of both metabolome and the occurrence of specific bacterial phylotypes. In contrast to the increase in total faecal iron after PO administration of iron sulfate tablets, the total sulfur concentration did not increase. Oral administration studies of radioactive sulfate indicate a relatively high efficiency of intestinal sulfate absorption, reaching up to $80 \% .^{36}$ Even doses of up to $8 \mathrm{~g}$ anhydrous sodium sulfate per day resulted in 50-60\% absorption efficiency. ${ }^{37}$ Although the colon might contribute to total sulfate absorption, ${ }^{38}$ colonic accumulation of this terminal electron acceptor as growth-limiting substrate of sulfate-reducing bacteria would lead to an increased abundance in human faeces, including Desulfovibrio, Desulfomonas or Desulfobacter. ${ }^{39}$ However, sequence analysis in our study revealed low relative abundance and no changes in bacterial taxa associated with sulfate metabolism. Changes included higher signals for cholesterol, palmitate and phosphatidylglycerol in PO-IRT, accompanied by a significant decrease in the relative abundance of $C$. aerofaciens, F. prausnitzii, $R$. bromii and Dorea sp., and an increase in one OTU within the genus Bifidobacterium. In contrast to the increase in total faecal iron after PO administration of iron sulfate tablets, the total sulfur 
concentration did not increase. Oral administration studies of radioactive sulfate indicate a relatively high efficiency of intestinal sulfate absorption, reaching up to $80 \%{ }^{36}$ Even doses of up to $8 \mathrm{~g}$ anhydrous sodium sulfate per day resulted in $50-60 \%$ absorption efficiency. ${ }^{37}$ Although the colon might contribute to total sulfate absorption, ${ }^{38}$ colonic accumulation of this terminal electron acceptor as growth-limiting substrate of sulfate-reducing bacteria would lead to an increased abundance in human faeces, including Desulfovibrio, Desulfomonas or Desulfobacter. ${ }^{39}$ However, sequence analysis in our study revealed low relative abundance and no changes in bacterial taxa associated with sulfate metabolism. Opposite to the IBD-related changes that we observed, iron fortification in Kenyan infants showed an increase in clostridia and a decrease in bifidobacteria. ${ }^{9}{ }^{40}$ Interestingly, Bifidobacterium strains isolated from stools of iron-deficient infants efficiently sequestered iron, ${ }^{41}$ suggesting an inverse relationship between abundance and functionality. Moreover, we cannot exclude the possibility that the intake of functional foods, including probiotic dairy products, in the present study may have specifically affected results on relative sequence abundances of bifidobacteria. Considering currently available studies, one of the most consistent differences between IBD and NI subjects is the reduced abundance of $F$. prausnitzii, a member of the Clostridium cluster IV. $^{1415}$ We demonstrated that the relative sequence abundance of $F$. prausnitzii in stool samples was lower after PO therapy, suggesting that this bacterium is affected not only by disease-intrinsic factors, but also by environmental factors.

Non-targeted metabolomics studies have described IBD-related changes in faecal metabolomes using mass spectrometry (MS)-based or nuclear magnetic resonance spectroscopy (NMR)-based measurements. ${ }^{42-47}$ We applied direct infusion FT-ICR-mass spectrometry (MS) to identify differences in the metabolic landscape of patients with IBD before and after PO-IRT or IV-IRT. Our models revealed best discrimination of faecal samples from CD versus NI subjects at baseline, with, for instance, decreased intensities for the metabolite pyridoxate, a degradation product of vitamin $\mathrm{B}_{6}$, which, in its coenzyme form (pyridoxal-5-phosphate), previously showed lower plasma levels in patients with $\mathrm{IBD}^{48}$ and was found to be inversely associated with systemic inflammatory biomarkers. ${ }^{49}$ Several other compounds annotated as fatty acids or conjugates thereof were discriminative between NI and IBD (both CD and UC) subjects, supporting a previously published study. ${ }^{45}$ Fatty acids are important players in mechanisms underlying inflammatory responses, particularly short chain fatty acids (SCFA) and polyunsaturated fatty acids. ${ }^{50}$ C18:0 was characteristic of CD samples, whereas signals for C16:2 were higher in the NI group. Of note, C16:2 can be produced from chain-shortening of conjugated linoleic acid, the latter compound being shown to have positive immunoregulatory effects in patients with CD. ${ }^{51} 52$ Phospholipids were also discriminatory in our analyses: lysophosphatidylethanolamine (LysoPA) was increased in $\mathrm{CD}$ and LysoPE was decreased in UC. LysoPAs can originate from ceramides, which are lipids regulating multiple cellular functions, including inflammation. ${ }^{53}$ Phosphorylation of ceramide leads to generation of ceramide phosphate that, along with LysoPAs, was increased in CD group compared with NI. With respect to UC subjects, the model revealed variations in the occurrence of dicarboxylic acids, such as undecanedioate, dodecanedioate and sebacate, which are proposed to regulate mitochondrial fatty acid oxidation and to be involved in IBD-related liver dysfunctions. ${ }^{54}$ Finally, intensities of acetyl-N-formyl-5methoxykynurenamine were slightly decreased in UC subjects at baseline. This compound is a metabolite of melatonin proposed to have anti-inflammatory properties. ${ }^{55}$ Data suggest that melatonin administration can have beneficial effects in UC. ${ }^{56}$

Metabolite features discriminating the PO and IV routes of IRT included cholesterol and bile acid derivatives (lower in PO). Different metabolic and inflammatory pathways involving cholesterol and bile acids have been linked to alterations of the gut microbiota. ${ }^{57-59}$ In addition, higher levels of C16:0 in the PO group were observed in our study. Patients with IBD were previously shown to have higher levels of saturated fatty acids in the colonic mucosa ${ }^{60}$ and patients with CD showed higher levels of C16:0 in faeces. ${ }^{45}$ Metabolites characterised by higher levels in the IV group included lysophosphatidic acid, a signalling molecule affecting different cell functions in the intestinal epithelium with possible tumorigenic effects, ${ }^{61}$ and urolithin $\mathrm{A}$, a metabolite of ellagic acid or ellagitannins produced by some members of the gut microbiota with a wide range of biological properties, for example, antioxidant, oestrogenic, antiinflammatory and anticarcinogenic activities. ${ }^{62-64}$ Altogether, whereas differences in microbiota were observed at the level of a few specific molecular species, the route of iron administration had a clearer discriminative effect at the level of metabolomes, which reflect both host and microbial functions.

In conclusion, IRT in patients with iron deficiency or anaemia induced significant shifts in bacterial community structure and metabolite landscape in faeces. Patients with CD seemed to be more prone to IRT-induced shifts and, according to the load of free iron detected in the luminal environment, oral iron therapy affected the presence of specific molecular bacterial species.

\section{Author affiliations}

'Division of Gastroenterology, Department of Medicine, University of Alberta, Edmonton, Canada

${ }^{2}$ Department of Gastroenterology, Wollongong Hospital, Wollongong, NSW, Australia ${ }^{3}$ ZIEL Institute for Food and Health, Technische Universität München, Freising, Germany

${ }^{4}$ Research Unit Analytical BioGeoChemistry, German Research Center for Environmental Health, Neuherberg, Germany

${ }^{5}$ Chair of Nutrition and Immunology, Technische Universität München, Freising, Germany

Acknowledgements The authors are grateful to Sabrina Cabric for technical assistance with sample preparation for high-throughput sequencing.

Contributors RF and DH designed the study. TL, TC, KS and AS performed the study and the experiments. KS, AW, ML and PS-K carried out metabolomics analysis. TC and IL analysed sequencing data. TL, TC, KS and DH wrote the manuscript.

Competing interests None declared.

\section{Patient consent Obtained.}

Ethics approval The study was approved by the local ethics committee of the University of Alberta (Canada) and registered with clinicaltrial.gov (NCT01067547),

Provenance and peer review Not commissioned; externally peer reviewed.

Open Access This is an Open Access article distributed in accordance with the Creative Commons Attribution Non Commercial (CC BY-NC 4.0) license, which permits others to distribute, remix, adapt, build upon this work non-commercially, and license their derivative works on different terms, provided the original work is properly cited and the use is non-commercial. See: http://creativecommons.org/ licenses/by-nc/4.0/

\section{REFERENCES}

1 Avni T, Bieber A, Steinmetz T, et al. Treatment of anemia in inflammatory bowel disease — systematic review and meta-analysis. PLOS ONE 2013;8:e75540.

2 Lee TW, Kolber MR, Fedorak RN, et al. Iron replacement therapy in inflammatory bowel disease patients with iron deficiency anemia: a systematic review and meta-analysis. J Crohns Colitis 2012;6:267-75.

3 Rizvi S, Schoen RE. Supplementation with oral vs. intravenous iron for anemia with IBD or gastrointestinal bleeding: is oral iron getting a bad rap? Am J Gastroenterol 2011;106:1872-9.

4 Kulnigg S, Stoinov S, Simanenkov V, et al. A novel intravenous iron formulation for treatment of anemia in inflammatory bowel disease: the ferric carboxymaltose (FERINJECT) randomized controlled trial. Am J Gastroenterol 2008;103:1182-92. 
5 Cassat JE, Skaar EP. Iron in infection and immunity. Cell Host Microbe 2013;13:509-19.

6 Payne SM, Wyckoff EE, Murphy ER, et al. Iron and pathogenesis of Shigella: iron acquisition in the intracellular environment. Biometals 2006;19:173-80.

7 Wyckoff EE, Mey AR, Leimbach A, et al. Characterization of ferric and ferrous iron transport systems in Vibrio cholerae. J Bacteriol 2006;188:6515-23.

8 Zimmermann MB, Chassard C, Rohner F, et al. The effects of iron fortification on the gut microbiota in African children: a randomized controlled trial in Cote d'Ivoire. Am J Clin Nutr 2010;92:1406-15.

9 Jaeggi T, Kortman GA, Moretti $D$, et al. Iron fortification adversely affects the gut microbiome, increases pathogen abundance and induces intestinal inflammation in Kenyan infants. Gut 2015;64:731-42.

10 Knights D, Lassen $K G$, Xavier RJ. Advances in inflammatory bowel disease pathogenesis: linking host genetics and the microbiome. Gut 2013;62:1505-10.

11 Rutgeerts $\mathrm{P}$, Goboes K, Peeters $\mathrm{M}$, et al. Effect of faecal stream diversion on recurrence of Crohn's disease in the neoterminal ileum. Lancet 1991;338:771-4.

12 Winslet MC, Allan A, Poxon V, et al. Faecal diversion for Crohn's colitis: a model to study the role of the faecal stream in the inflammatory process. Gut 1994:35:236-42.

13 Gruber L, Lichti P, Rath E, et al. Nutrigenomics and nutrigenetics in inflammatory bowel diseases. J Clin Gastroenterol 2012:46:735-47.

14 Machiels K, Joossens M, Sabino J, et al. A decrease of the butyrate-producing species Roseburia hominis and Faecalibacterium prausnitzii defines dysbiosis in patients with ulcerative colitis. Gut 2014:63:1275-83.

15 Sokol H, Seksik P, Furet JP, et al. Low counts of Faecalibacterium prausnitzii in colitis microbiota. Inflamm Bowel Dis 2009:15:1183-9.

16 Walker AW, Sanderson JD, Churcher C, et al. High-throughput clone library analysis of the mucosa-associated microbiota reveals dysbiosis and differences between inflamed and non-inflamed regions of the intestine in inflammatory bowel disease. BMC Microbiol 2011:11:7.

17 Werner T, Wagner SJ, Martínez I, et al. Depletion of luminal iron alters the gut microbiota and prevents Crohn's disease-like ileitis. Gut 2011;60:325-33.

18 Weiss $\mathrm{G}$. Dietary iron supplementation: a proinflammatory attack on the intestine? Gut 2015;64:696-7.

19 Lagkouvardos I, Kläering K, Heinzmann SS, et al. Gut metabolites and bacteria community networks during a pilot intervention study with flaxseeds in healthy adult men. Mol Nutr Food Res 2015;59:1614-28.

20 Goldberg ND. Iron deficiency anemia in patients with inflammatory bowel disease. Clin Exp Gastroenterol 2013:6:61-70

21 Tolkien Z, Stecher L, Mander AP, et al. Ferrous sulfate supplementation causes significant gastrointestinal side-effects in adults: a systematic review and meta-analysis. PLOS ONE 2015;10:e117383.

22 Gomollón F, Gisbert JP. Intravenous iron in inflammatory bowel diseases. Curr Opin Gastroenterol 2013;29:201-7.

23 Kulnigg S, Gasche C. Systematic review: managing anaemia in Crohn's disease. Aliment Pharmacol Ther 2006;24:1507-23.

24 Werner T, Hoermannsperger G, Schuemann K, et al. Intestinal epithelial cell proteome from wild-type and TNFDeltaARE/WT mice: effect of iron on the development of chronic ileitis. J Proteome Res 2009;8:3252-64.

25 Gevers D, Kugathasan S, Denson LA, et al. The treatment-naive microbiome in new-onset Crohn's disease. Cell Host Microbe 2014;15:382-92.

26 Lepage P, Häsler R, Spehlmann ME, et al. Twin study indicates loss of interaction between microbiota and mucosa of patients with ulcerative colitis. Gastroenterology 2011:141:227-36.

27 Manichanh C, Rigottier-Gois L, Bonnaud E, et al. Reduced diversity of faecal microbiota in Crohn's disease revealed by a metagenomic approach. Gut 2006:55:205-11.

28 Willing BP, Dicksved J, Halfvarson J, et al. A pyrosequencing study in twins shows that gastrointestinal microbial profiles vary with inflammatory bowel disease phenotypes. Gastroenterology 2010;139:1844-54.e1.

29 Momozawa Y, Deffontaine V, Louis $\mathrm{E}$, et al. Characterization of bacteria in biopsies of colon and stools by high throughput sequencing of the V2 region of bacterial 16S rRNA gene in human. PLOS ONE 2011;6:e16952.

30 Andoh A, Tsujikawa T, Sasaki M, et al. Faecal microbiota profile of Crohn's disease determined by terminal restriction fragment length polymorphism analysis. Aliment Pharmacol Ther 2009:29:75-82.

31 Joossens $\mathrm{M}$, Huys $\mathrm{G}$, Cnockaert $\mathrm{M}$, et al. Dysbiosis of the faecal microbiota in patients with Crohn's disease and their unaffected relatives. Gut 2011;60:631-7.

32 Atarashi $\mathrm{K}$, Tanoue T, Oshima $\mathrm{K}$, et al. Treg induction by a rationally selected mixture of Clostridia strains from the human microbiota. Nature 2013:500:232-6.

33 Morotomi M, Nagai F, Watanabe Y. Description of Christensenella minuta gen. nov., sp. nov., isolated from human faeces, which forms a distinct branch in the order Clostridiales, and proposal of Christensenellaceae fam. nov. Int I Syst Evol Microbiol 2012;62:144-9.

34 Png CW, Lindén SK, Gilshenan KS, et al. Mucolytic bacteria with increased prevalence in IBD mucosa augment in vitro utilization of mucin by other bacteria. Am J Gastroenterol 2010;105:2420-8.
35 Wills ES, Jonkers DM, Savelkoul PH, et al. Fecal microbial composition of ulcerative colitis and Crohn's disease patients in remission and subsequent exacerbation. PLOS ONE 2014;9:e90981.

36 Bauer JH. Oral administration of radioactive sulfate to measure extracellular fluid space in man. J Appl Physiol 1976;40:648-50.

37 Cocchetto DM, Levy G. Absorption of orally administered sodium sulfate in humans. J Pharm Sci 1981;70:331-3.

38 Florin T, Neale G, Gibson GR, et al. Metabolism of dietary sulphate: absorption and excretion in humans. Gut 1991;32:766-73.

39 Beerens $\mathrm{H}$, Romond C. Sulfate-reducing anaerobic bacteria in human feces. Am J Clin Nutr 1977:30:1770-6.

40 Krebs NF, Sherlock LG, Westcott J, et al. Effects of different complementary feeding regimens on iron status and enteric microbiota in breastfed infants. J Pediatr 2013;163:416-23

41 Vazquez-Gutierrez P, Lacroix C, Jaeggi T, et al. Bifidobacteria strains isolated from stools of iron deficient infants can efficiently sequester iron. BMC Microbiol 2015;15:3.

42 Ahmed I, Greenwood R, Costello Bde L, et al. An investigation of fecal volatile organic metabolites in irritable bowel syndrome. PLOS ONE 2013:8:e58204.

43 Bjerrum JT, Wang Y, Hao F, et al. Metabonomics of human fecal extracts characterize ulcerative colitis, Crohn's disease and healthy individuals. Metabolomics 2015;11:122-33

44 Gao X, Pujos-Guillot E, Martin JF, et al. Metabolite analysis of human fecal water by gas chromatography/mass spectrometry with ethyl chloroformate derivatization. Anal Biochem 2009;393:163-75

45 Jansson J, Willing B, Lucio $\mathrm{M}$, et al. Metabolomics reveals metabolic biomarkers of Crohn's disease. PLOS ONE 2009;4:e6386.

46 Le Gall G, Noor SO, Ridgway K, et al. Metabolomics of fecal extracts detects altered metabolic activity of gut microbiota in ulcerative colitis and irritable bowel syndrome. J Proteome Res 2011;10:4208-18.

47 Marchesi JR, Holmes E, Khan F, et al. Rapid and noninvasive metabonomic characterization of inflammatory bowel disease. J Proteome Res 2007;6:546-51.

48 Saibeni S, Cattaneo M, Vecchi $M$, et al. Low vitamin B(6) plasma levels, a risk factor for thrombosis, in inflammatory bowel disease: role of inflammation and correlation with acute phase reactants. Am J Gastroenterol 2003:98:112-17.

49 Sakakeeny L, Roubenoff R, Obin M, et al. Plasma pyridoxal-5-phosphate is inversely associated with systemic markers of inflammation in a population of US adults. J Nutr 2012;142:1280-5.

50 Heimerl S, Moehle C, Zahn A, et al. Alterations in intestinal fatty acid metabolism in inflammatory bowel disease. Biochim Biophys Acta 2006;1762:341-50.

51 Bassaganya-Riera J, Hontecillas R, Horne WT, et al. Conjugated linoleic acid modulates immune responses in patients with mild to moderately active Crohn's disease. Clin Nutr 2012;31:721-7.

52 Müller A, Ringseis R, Düsterloh K, et al. Detection of conjugated dienoic fatty acids in human vascular smooth muscle cells treated with conjugated linoleic acid. Biochim Biophys Acta 2005:1737:145-51.

53 Arana L, Gangoiti P, Ouro A, et al. Ceramide and ceramide 1-phosphate in health and disease. Lipids Health Dis 2010:9:15.

54 Uko V, Thangada S, Radhakrishnan K. Liver disorders in inflammatory bowel disease. Gastroenterol Res Pract 2012;2012:642923.

55 Mayo JC, Sainz RM, Tan DX, et al. Anti-inflammatory actions of melatonin and its metabolites, N1-acetyl-N2-formyl-5-methoxykynuramine (AFMK) and N1-acetyl-5-methoxykynuramine (AMK), in macrophages. J Neuroimmunol 2005; 165:139-49.

56 Terry PD, Villinger F, Bubenik GA, et al. Melatonin and ulcerative colitis: evidence, biological mechanisms, and future research. Inflamm Bowel Dis 2009;15:134-40.

57 Devkota S, Wang Y, Musch MW, et al. Dietary-fat-induced taurocholic acid promotes pathobiont expansion and colitis in II10-/- mice. Nature 2012;487:104-8.

58 Duboc H, Rajca S, Rainteau D, et al. Connecting dysbiosis, bile-acid dysmetabolism and gut inflammation in inflammatory bowel diseases. Gut 2013;62:531-9.

59 Gerrard P. Metabolism of cholesterol and bile acids by the gut microbiota. Pathogens 2013;3:14-24.

60 Fernández-Bañares F, Esteve-Comas M, Mañé J, et al. Changes in mucosal fatty acid profile in inflammatory bowel disease and in experimental colitis: a common response to bowel inflammation. Clin Nutr 1997;16:177-83.

61 Yun CC, Kumar A. Diverse roles of LPA signaling in the intestinal epithelium. Exp Cell Res 2015;333:201-7.

62 Espín JC, Larrosa M, García-Conesa MT, et al. Biological significance of urolithins, the gut microbial ellagic acid-derived metabolites: the evidence so far. Evid Based Complement Alternat Med 2013:2013:270418.

63 Selma MV, Tomás-Barberán FA, Beltrán D, et al. Gordonibacter urolithinfaciens sp. nov., a urolithin-producing bacterium isolated from the human gut. Int I Syst Evol Microbiol 2014:64:2346-52.

64 Tomás-Barberán FA, García-Villalba R, González-Sarrias A, et al. Ellagic acid metabolism by human gut microbiota: consistent observation of three urolithin phenotypes in intervention trials, independent of food source, age, and health status. J Agric Food Chem 2014;62:6535-8. 